\title{
Superresolved femtosecond laser nanosurgery of cells
}

\author{
Matthias Pospiech $^{1}$, Moritz Emons ${ }^{1}$, Kai Kuetemeyer ${ }^{2}$, Alexander \\ Heisterkamp ${ }^{2}$, and Uwe Morgner ${ }^{1,2}$ \\ ${ }^{1}$ Institute of Quantum Optics, Leibniz Universität Hannover, Welfengarten 1, D-30167 \\ Hannover, Germany \\ ${ }^{2}$ Laser Zentrum Hannover e.V., Hollerithallee 8, D-30419 Hannover, Germany \\ pospiech@iqo.uni-hannover.de
}

\begin{abstract}
We report on femtosecond nanosurgery of fluorescently labeled structures in cells with a spatially superresolved laser beam. The focal spot width is reduced using phase filtering applied with a programmable phase modulator. A comprehensive statistical analysis of the resulting cuts demonstrates an achievable average resolution enhancement of $30 \%$.
\end{abstract}

(C) 2011 Optical Society of America

OCIS codes: (100.6640) Superresolution, (350.5730) Resolution, (170.0180) Microscopy, (180.2520) Fluorescence microscopy, (070.6120) Spatial light modulators, (140.7090) Ultrafast lasers

\section{References and links}

1. W. Denk, J. Strickler, and W. Webb, “Two-photon laser scanning fluorescence microscopy,” Science 248, 73-76 (1990).

2. W. R. Zipfel, R. M. Williams, and W. W. Webb, "Nonlinear magic: multiphoton microscopy in the biosciences," Nat Biotech 21, 1369-1377 (2003).

3. K. König, I. Riemann, and W. Fritzsche, "Nanodissection of human chromosomes with near-infrared femtosecond laser pulses," Opt. Lett. 26, 819-821 (2001).

4. T. Shimada, W. Watanabe, S. Matsunaga, T. Higashi, H. Ishii, K. Fukui, K. Isobe, and K. Itoh, "Intracellular disruption of mitochondria in a living hela cell with a 76-mhz femtosecond laser oscillator," Opt. Express 13, 9869-9880 (2005).

5. L. Sacconi, I. M. T.-N. rrelykke, R. Antolini, and F. S. Pavone, "Combined intracellular three-dimensional imaging and selective nanosurgery by a nonlinear microscope," Journal of Biomedical Optics 10, 014002 (2005).

6. A. Heisterkamp, I. Z. Maxwell, E. Mazur, J. M. Underwood, J. A. Nickerson, S. Kumar, and D. E. Ingber, "Pulse energy dependence of subcellular dissection by femtosecond laser pulses," Opt. Express 13, 3690-3696 (2005).

7. A. Vogel, J. Noack, G. Hüttman, and G. Paltauf, "Mechanisms of femtosecond laser nanosurgery of cells and tissues," Applied Physics B: Lasers and Optics 81, 1015-1047 (2005). 10.1007/s00340-005-2036-6.

8. K. Kuetemeyer, R. Rezgui, H. Lubatschowski, and A. Heisterkamp, "Influence of laser parameters and staining on femtosecond laser-based intracellular nanosurgery," Biomed. Opt. Express 1, 587-597 (2010).

9. T. R. M. Sales and G. M. Morris, "Fundamental limits of optical superresolution," Opt. Lett. 22, 582-584 (1997).

10. G. Boyer, "New class of axially apodizing filters for confocal scanning microscopy," J. Opt. Soc. Am. A 19, 584-589 (2002).

11. D. M. de Juana, J. E. Oti, V. F. Canales, and M. P. Cagigal, "Transverse or axial superresolution in a 4pi-confocal microscope by phase-only filters," J. Opt. Soc. Am. A 20, 2172-2178 (2003).

12. P. N. Gundu, E. Hack, and P. Rastogi, "Apodized superresolution - concept and simulations," Optics Communications 249, $101-107$ (2005).

13. I. J. Cox, "Increasing the bit packing densities of optical disk systems," Appl. Opt. 23, 3260-3261 (1984).

14. M. Shinoda and K. Kime, "Focusing characteristics of an optical head with superresolution using a high-aspectratio red laser diode," Japanese Journal of Applied Physics 35, 380-383 (1996).

15. M. R. Wang and X. G. Huang, "Subwavelength-resolvable focused non-gaussian beam shaped with a binary diffractive optical element,” Appl. Opt. 38, 2171-2176 (1999).

\#137728 - \$15.00 USD Received 4 Nov 2010; revised 21 Dec 2010; accepted 22 Dec 2010; published 5 Jan 2011

(C) 2011 OSA 1 February 2011 / Vol. 2, No. 2 / BIOMEDICAL OPTICS EXPRESS 264 
16. H. Ando, "Phase-shifting apodizer of three or more portions," Japanese Journal of Applied Physics 31, 557-567 (1992).

17. H. Wang and F. Gan, "High focal depth with a pure-phase apodizer," Appl. Opt. 40, 5658-5662 (2001).

18. de Juana, V. F. Canales, P. J. Valle, and M. P. Cagigal, "Focusing properties of annular binary phase filters," Optics Communications 229, 71-77 (2004).

19. H. Liu, Y. Yan, and G. Jin, "Design and experimental test of diffractive superresolution elements," Appl. Opt. 45, 95-99 (2006).

20. L. Liu, F. Diaz, L. Wang, B. Loiseaux, J.-P. Huignard, C. J. R. Sheppard, and N. Chen, "Superresolution along extended depth of focus with binary-phase filters for the gaussian beam," J. Opt. Soc. Am. A 25, 2095-2101 (2008).

21. P. A. Quinto-Su and V. Venugopalan, "Mechanisms of laser cellular microsurgery," in "Laser Manipulation of Cells and Tissues,", vol. 82 of Methods in Cell Biology, M. W. Berns and K. O. Greulich, eds. (Academic Press, 2007), pp. 111, $113-151$.

\section{Introduction}

Multiphoton fluorescence microscopy based on femtosecond laser scanning [1,2] is a powerful technique for three dimensional optical sectioning in life sciences. The method is based on the simultaneous absorption of two or three photons in the focal volume of a high NA microscope objective. The same setup is suited for nanodissection of living cells and subcellular structures [3-6]. This destructive manipulation occurs if the energy per pulse exceeds a certain threshold and induces nonlinear multiphoton absorption and avalanche ionization. In the case of $\mathrm{MHz}$ repetition rate laser systems, as used in this work, the free electrons generate a low-density plasma in the focal region inducing photochemical effects (e.g. bond breaking of biomolecules). Accumulation over many pulses leads to dissection of biological material. In this regime, no significant heat or mechanical energy transfer to surrounding regions occurs $[7,8]$. A very small lateral extend of the modified focal volume is required to minimize collateral damage in the vicinity of the laser focus and to improve long-term cell viability. In addition, very precise dissection allows for the manipulation of single-cell organelles.

By using high NA microscope objectives and laser pulse energies close to the ablation threshold, the lateral extend of the modified material is limited to less than $1 \mu \mathrm{m}[3,7]$. This diffractionlimited resolution can be further improved by techniques generally referred to as superresolution [9]. Superresolution is achieved by controlling the phase and/or amplitude of the laser beam and has shown considerable impact for many applications such as confocal scanning microscopy [10-12] or optical data storage [13-15]. In this case, a diffractive filter is placed at the exit pupil of an optical system, as depicted in figure 1.

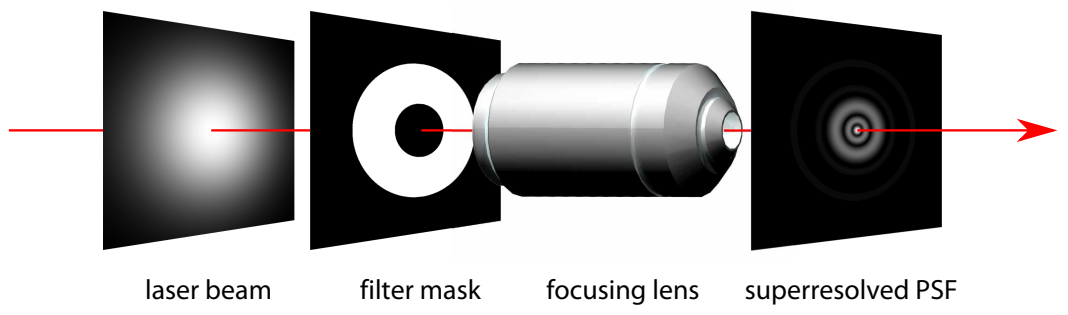

Fig. 1. Beam propagation through filter and focusing lens.

In the first approaches, these filters were realized by obstruction of light in concentric annular zones [13]. Later, attention was shifted to the design of phase-only pupil filters [16-20] due to their better transmittance. These phase filters have been based on two or more annular zones with a mutual phase difference of $\pi$ radians. The spot size reduction is typically accompanied by an extended depth of focus $[17,20]$. These phase-only filters can be implemented dynamically 
in optical instruments by use of a phase-controlling device such as a deformable mirror or a Spatial Light Modulator (SLM). In addition, it is possible to produce these phase masks as static phase filters in glass $[15,19]$.

The improvement achieved in spot size was on the order of $30 \%$. However, to the best of our knowledge, this technique has so far not been investigated with respect to the nanosurgery of cells, which we demonstrate here for the first time.

\section{Design of diffractive superresolution filter}

We are working with phase-only filters consisting of three annular zones with a phase difference of $\pi$ radians between adjacent zones, as displayed in figure 2a. The filter is defined by the radii $r_{a}$ normalized to the maximum aperture and $r_{b}$ with $0 \leq r_{a} \leq r_{b} \leq 1$ and $\Delta r=r_{b}-r_{a}$ which is then reimaged onto the exit pupil of the microscope objective.

The performance of any superresolution filter is typically described by the point spread function (PSF) in the focal plane. We define a normalized spot size $G$ as the radius at which the central peak intensity of a superresolved PSF falls down to the first zero divided by the corresponding radius of the unshaped Airy disk pattern; furthermore, a Strehl ratio $S$ as the central intensity of a superresolved PSF divided by that of the unshaped Airy disk pattern; and finally a normalized side lobe quantifier $M$ as the intensity of the highest side lobe divided by the central intensity of the PSF [9]. Figure 2b illustrates the definition of the three figures for a typical superresolved PSF. Lower $G$, higher $S$, and lower $M$ values are favorable for high resolution performance.
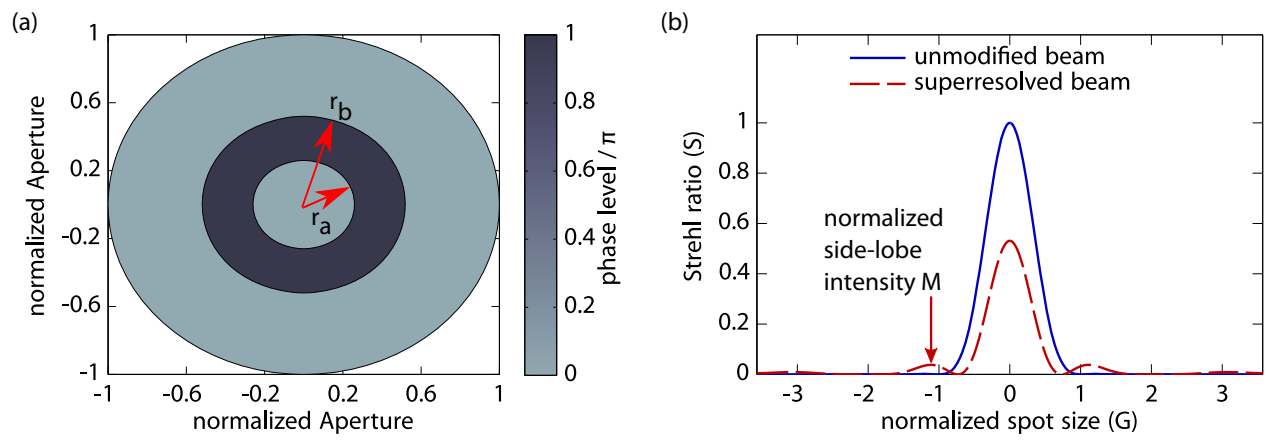

Fig. 2. (a) Definition of phase filter radii. (b) Example point spread function of an unmodified beam and of a superresolved beam.

Figure 3a shows the calculated PSF for increasing $\Delta r$; the extracted performance factors are pictured in figure $3 \mathrm{~b}$. The normalized spot size (G) as well as the Strehl ratio (S) decrease almost linearly, however, the Strehl ratio decreases at a faster rate. For example at $\Delta r=0.3$ the spot size decreases to $50 \%$, however at the cost of a Strehl ratio of 0.14 and a side lobe intensity of 0.55 . For $\Delta r$ increasing from 0 to 0.3 the depth of focus is increasing by $40 \%$. 

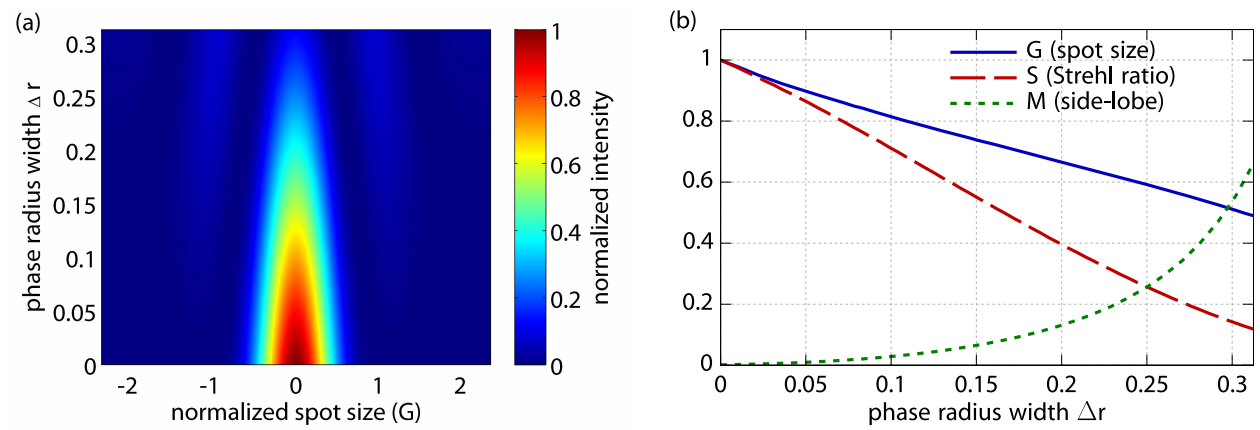

Fig. 3. Left: Evolution of the point spread function with increasing phase ring width $\Delta r$ for $r_{a}=0.16$. Right: Corresponding superresolution performance factors.

\section{Experimental setup}

The laser system is a home-built femtosecond Yb:KYW laser oscillator operating at $1030 \mathrm{~nm}$ with a repetition rate of $44 \mathrm{MHz}$ and up to $10 \mathrm{~nJ}$ of pulse energy at $240 \mathrm{fs}$ pulse duration. The experimental setup is shown in figure 4 .

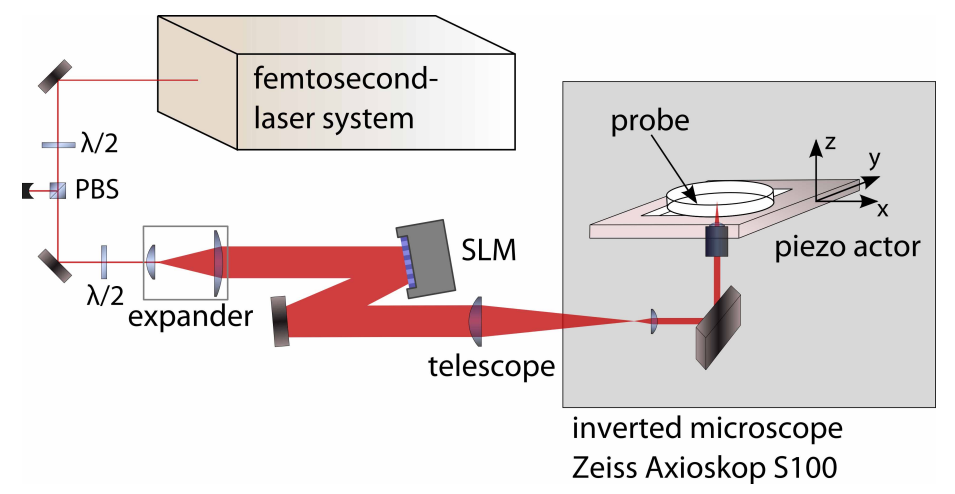

Fig. 4. Experimental setup for generation of superresolved beams and subsequent use in nanosurgery of biological probes.

The phase filter is integrated into the beam by a reflective SLM, here a liquid-crystal phase modulator (Hamamatsu PPM, model X8267-15). The SLM consists of an array of $768 \times 768$ pixels with a pixel size of $\approx 26 \mu \mathrm{m}$, addressed via computer control. The incident power can be varied by a $\lambda / 2$-plate and a polarizing beam splitter (PBS); the beam is expanded to a Gaussian radius of $8 \mathrm{~mm}$ so that the $20 \mathrm{~mm} \times 20 \mathrm{~mm}$ aperture of the SLM is almost filled. The SLM is inserted under a small angle, and the shaped laser beam is demagnified by a second telescope to match the aperture of the final microscope objective.

We measured the superresolution performance figures in the focal plane of a $500 \mathrm{~mm}$ lens using a CMOS Camera (Sumix SMX150). Figure 5 demonstrates a typical superresolved PSF measured with the camera (graph (a) and (b)) in comparison with the corresponding simulated superresolved PSF (c). The superresolved imaged beam reveals a small deviation from circular symmetry due to a residual beam ellipticity of $20 \%$. This originates directly from the laser output which reveals the same ellipticity. The evaluation of the Strehl ratio $S$ and the width $G$ is performed along the center of the beam in $x$-direction. 

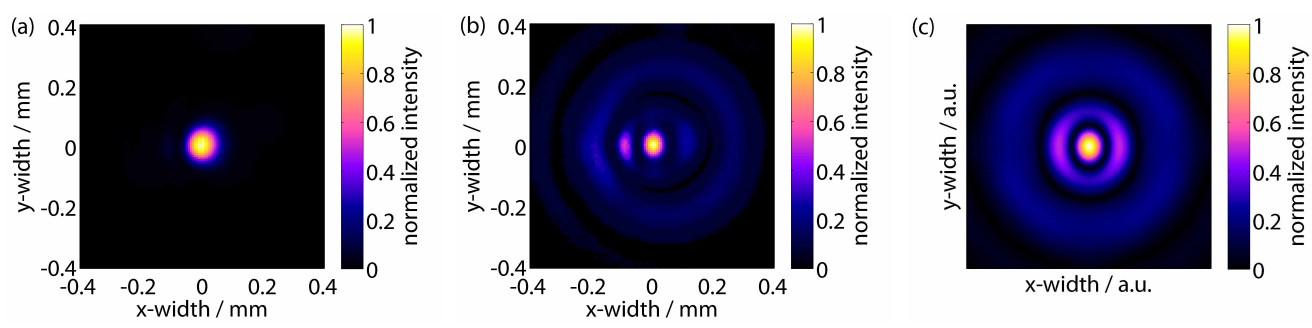

Fig. 5. Normalized camera images in the focal plane of a $500 \mathrm{~mm}$ lens showing an Gaussian beam (a) and a superresolved beam (b) with a phase ring width of $\Delta r=0.2$. (c) Calculated superresolved beam with the same parameters as (b).

The resulting graphs for peak intensity (Strehl ratio $S$ ) and the spot width $(G)$ over the phase ring width are displayed in figure 6 . The blue lines are from a linear fit. For $20 \%$ reduction in spot size the peak intensity decreases by $40 \%\left(\Delta r=0.1, r_{a}=0.16\right)$, and for $30 \%$ reduction the intensity decreases by $60 \%\left(\Delta r=0.16, r_{a}=0.16\right)$. These dependencies match the theoretical calculations from figure $6 \mathrm{~b}$ very well.
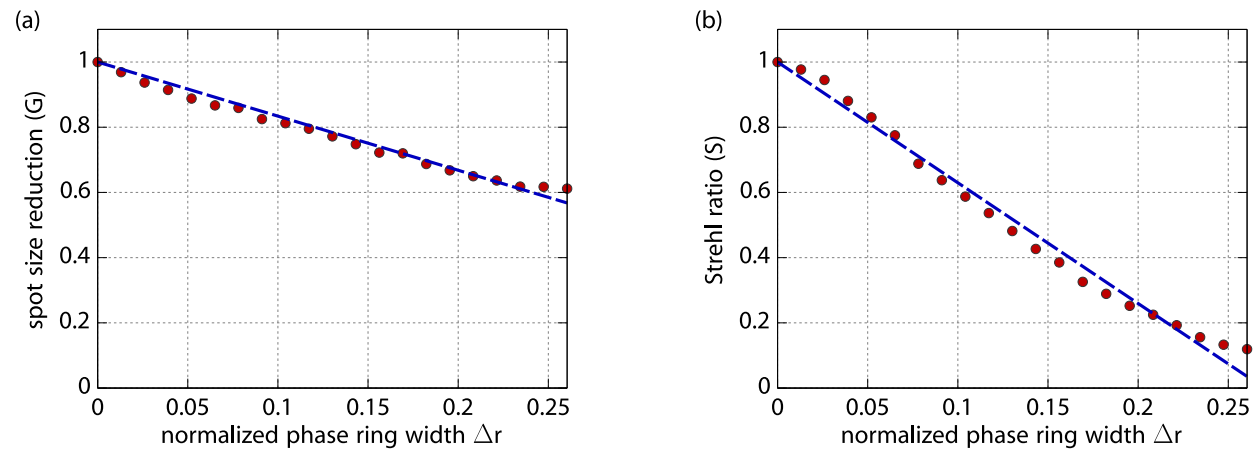

Fig. 6. Reduction in spot width (G) over phase radius $\Delta r$ (a) and Strehl ratio (S) over phase radius $\Delta r$ (b) for $r_{a}=0.16$. The dashed blue lines show the linear regression.

\section{Nanosurgery of cells}

The implication of superresolved beams on the width of femtosecond laser-based nanosurgery of cells was analyzed using line cuts in labeled bovine bovine capillary endothelial cells. These cells were cultivated in RPMI 1640 medium (Roswell Park Memorial Institute) supplemented with $10 \%$ FCS (fetal calf serum) and the antibiotics penicillin, streptomycin, and partricin at $37^{\circ} \mathrm{C}$ and $5 \% \mathrm{CO}_{2}$ humidified atmosphere. For nanosurgery experiments, cells were fixed in $4 \%$ paraformaldehyde (Sigma Aldrich) in phosphate buffered saline (PBS) for 20 minutes and nuclear DNA was stained with Hoechst 33342 (Invitrogen) for 10 minutes.

The sample was mounted on a piezo controlled scanning unit based on a 3D piezo actor (TRITOR 100, Piezo Jena) placed in an inverted microscope (Zeiss Axiovert S100) as depicted in figure 4. Cell nanodissection was done by moving the probe with respect to the fixed laser focus at a moving speed of $100 \mu \mathrm{m} / \mathrm{s}$. The laser was focused into the cells using a water immersion IR optimized 0.8 NA microscope objective (Zeiss Achroplan IR 40x/0.8W). The cells were tagged with fluorescence molecules to analyze the resulting structures in a multiphoton fluorescence microscope with a 100x/1.3 NA objective. The magnification of the cutting objective has been chosen 2.5 times smaller than for microscopy to obtain clear measures of the cell cutting 
widths. Depending on the incident laser power, the fluorescence markers are photobleached or completely destroyed due to material ablation with a threshold about $20 \%$ higher for ablation than for bleaching. From the amount of fluorescence signal only, one could not discriminate between the two processes, but from [6] it is well known that the ablation threshold is reached for cutting widths corresponding to the focal spot size. To ensure true material cutting, the processing power was varied from $5.2 \mathrm{~nJ}$ well above the ablation threshold down towards $0.7 \mathrm{~nJ}$ with expected decreasing dissection width $[6,8]$.

In total, 105 cell cuts were done and analyzed both with an unmodified laser beam acting as the reference and with different superresolution filter functions; results with the same peak intensity taking the reduced Strehl ratio into account were compared. Examples are displayed in figure 7.
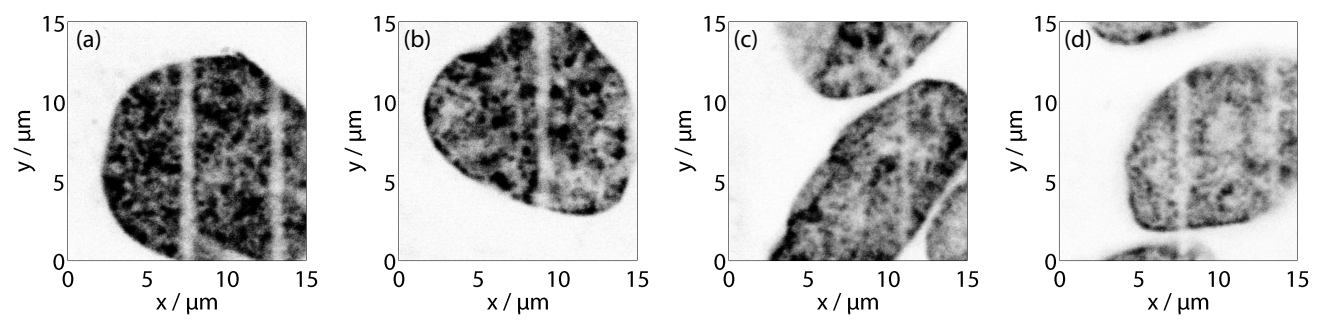

Fig. 7. Examples of line cuts in cells with an unshaped laser beam (a-b) and a superresolved laser beam (c-d) at $\Delta r=0.16$. The images a-d were obtained using a scanning multiphoton fluorescence microscope with a 100x/1.3 NA objective. The corresponding line width are derived as (a,1.) $1.09 \pm 0.18 \mu \mathrm{m}$ at $1.1 \mathrm{~nJ},(\mathrm{a}, 2$.) $1.09 \pm 0.33 \mu \mathrm{m}$ at $1.1 \mathrm{~nJ}$, (b) $0.87 \pm 0.22 \mu \mathrm{m}$ at $1.1 \mathrm{~nJ}$, (c) $0.76 \pm 0.25 \mu \mathrm{m}$ at $4.5 \mathrm{~nJ}$. (d,1.) $0.86 \pm 0.15 \mu \mathrm{m}$ at $4.3 \mathrm{~nJ}$. (d,2.) $1.07 \pm 0.30 \mu \mathrm{m}$ at $4.3 \mathrm{~nJ}$.

The modified cell structure was analyzed over the whole length by a Matlab script resulting in the width of the incision and a standard deviation. Since the fluorescence signal in the cell nuclei is not homogeneous, care was taken to analyze only the width of missing fluorescence signal due to removed material instead of missing signal due to natural inhomogeneities. Therefore each pixel line perpendicular to the cut direction was examined whether it contains suitable data for extraction of a width value at half maximum. In each line the intensity dip at the cut position was detected as a minimum between two adjacent local maxima and numbers have only been evaluated for sufficient contrast between the minimum and the maxima. The resulting data is plotted in figure 8. Three different cases are displayed: cell cuts with no superresolved beam, cuts with phase rings of width $\Delta r=0.1$, and with $\Delta r=0.16$. Since material processing is determined only by the maximum intensity of the central peak, the pulse energy axis includes the Strehl ratio of the PSF, which enables a direct comparison of the data resulting from unmodified and superresolved beams. The intensity of the side-lobes is well below the threshold for photo damage with no influence on the cutting width considering their relative intensity (M) of $7 \%$ at $\Delta r=0.16$ and the high nonlinearity of the process [7].

The smallest measured cutting widths are comparable to the spot diameter of the unshaped focal spot size $(0.78 \mu \mathrm{m})$ [21] indicating that true material ablation occurred over the whole intensity range [6]. 


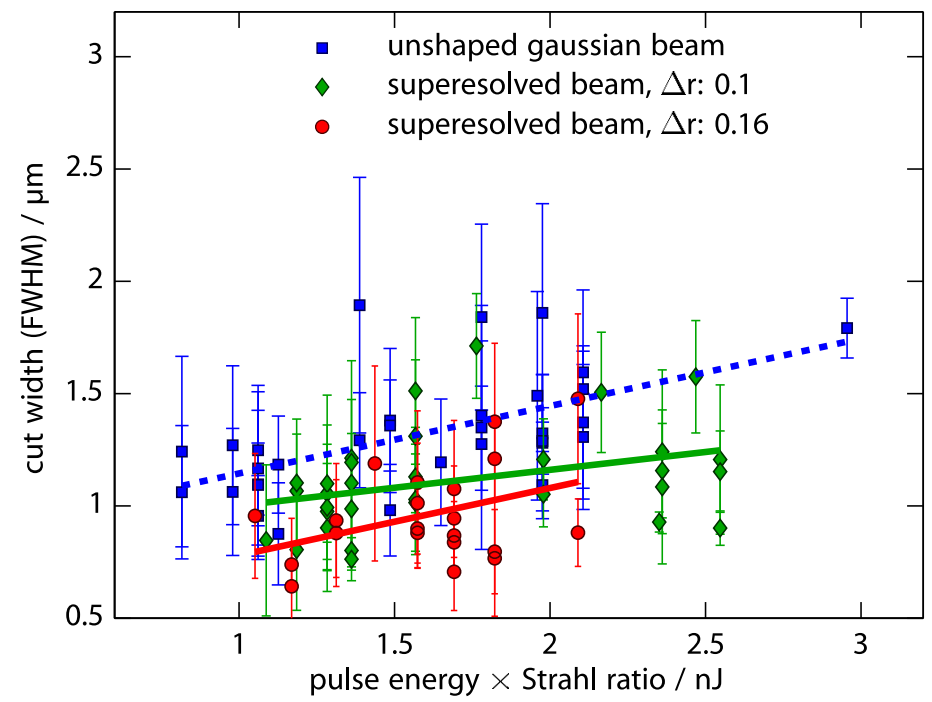

Fig. 8. Results of the statistical analysis of 105 cell cuts, divided into three sections: cell cuts with the reference beam (blue dashed line), cuts with $\Delta r=0.10$ (green solid line), and with $\Delta r=0.16$ (red solid line). The error bars represent the standard deviation of the analyzed width data. The pulse energy of the superresolved beams is normalized to the unshaped Strehl ratio.

A clear tendency between pulse energy and cutting width was visible. For decreasing energies, the resulting width decreased until a removal of material could not be clearly stated. This dependency could be identified for unmodified and for superresolved beams with the main difference that the medium width was considerably smaller over the whole range for superresolved beams. This reduction between the unmodified beam and the best superresolved beam $(\Delta r=0.16$, red) was about $30 \%$, which matched the measured spot width reduction (figure 5a) almost exactly.

\section{Conclusion}

We demonstrated for the first time the nanosurgery of cells with superresolved beams. The employed phase masks are based on three annular zones. The resulting incision width was decreased by $30 \%$ compared to an unmodified laser beam. Static phase masks allow for the same performance at considerably lower cost and dynamic phase masks, as implemented here, can be used to tune the spot width dynamically.

\section{Acknowledgments}

This work was funded by the German Federal Ministry for Education and Research (BMBF) under contract 13N9439 (Nanotome), as well as by the Deutsche Forschungsgemeinschaft (DFG, German Research Foundation) within the Cluster of Excellence 'REBIRTH' (From Regenerative Biology to Reconstructive Therapy). 